\title{
Incremental health care resource utilization and expenditures associated with autosomal-dominant polycystic kidney disease
}

This article was published in the following Dove Press journal:

ClinicoEconomics and Outcomes Research

\author{
Neeraj $\mathrm{N}$ lyer ${ }^{\prime}$ \\ Nicholas J Vendetti ${ }^{2}$ \\ Daniel I Levy ${ }^{3}$ \\ Jack Mardekian ${ }^{4}$ \\ Marko A Mychaskiw ${ }^{2}$ \\ Joseph Thomas III'
}

'Regenstrief Center for Healthcare Engineering and Center for Health Outcomes Research and Policy, College of Pharmacy, Purdue University, West Lafayette, IN, USA; ${ }^{2}$ Outcomes and Evidence, Pfizer Inc., Collegeville, PA, USA; ${ }^{3}$ Rare Disease Group, Global Product Development, Pfizer Inc., Collegeville, PA, USA; ${ }^{4}$ Biostatistics, Global Product Development, Pfizer Inc., Collegeville, PA, USA
Correspondence: Joseph Thomas III College of Pharmacy, Purdue University, Heine Pharmacy Building, Room 502A, 575 Stadium Mall Drive, West Lafayette, IN 47907-209I, USA

Tel + I $765494 \mid 477$

Fax +I 765496 I886

Email jt3@purdue.edu
Purpose: Incremental health care resource utilization and expenditures associated with autosomal dominant polycystic kidney disease (ADPKD) were estimated.

Methods: Study data were from a large administrative claims database. Individuals aged 18 years or older enrolled in tracked health plans for 12 months from April 1, 2011 through March 31, 2012, and with an International Classification of Disease, Ninth Revision, Clinical Modification diagnosis code for "polycystic kidney, autosomal dominant" (753.13) or for "polycystic kidney, unspecified type" (753.12) were identified as having ADPKD, and linked one-to-one with individuals without ADPKD based on age and gender. Zero-inflated negative binomial models estimated incremental health care resource utilization and expenditures, adjusting for risk factors. Results: A total of 3,844 individuals with ADPKD who satisfied selection criteria were linked one-to-one with 3,844 individuals without ADPKD. Multivariate, regression models adjusting for risk factors revealed incremental mean (standard error) resource use associated with ADPKD of 0.68 (0.090) hospital days, equal to 68 additional hospital days per 100 ADPKD patients, and 6.9 (0.28) outpatient visits, equal to 690 additional visits per 100 ADPKD patients. Mean (standard error) incremental total expenditures associated with ADPKD were US\$8,639 (\$470). Mean incremental expenditures were largest for outpatient expenditures at US\$4,918 (\$198), followed by mean incremental hospital expenditures of US\$2,603 (\$263), and mean incremental medication expenditures of US\$1,589 (\$77). Based on sub-group analysis, mean incremental total expenditures were US\$2,944 (\$417) among ADPKD patients without end-stage renal disease and US\$38,962 $(\$ 6,181)$ for those with end-stage renal disease.

Conclusion: ADPKD was associated with considerable incremental health care resource utilization and expenditures. Significant illness burden was found even before patients reached end-stage renal disease.

Keywords: ADPKD, economic impact, disease burden, health care resource use, health care costs

\section{Introduction}

Polycystic kidney disease (PKD) is a serious systemic disease that damages the kidneys and causes significant morbidity in patients' lives. ${ }^{1}$ PKD is characterized by development of multiple cysts with gradual kidney enlargement and other sequelae including hypertension, kidney hemorrhage, colonic diverticulitis, polycystic liver disease, intracranial aneurysms, and cysts in other tissues such as seminal vesicles, arachnoid membranes, and the pancreas. ${ }^{2-10}$ As cysts develop and grow in size, they impinge upon kidney parenchyma, ultimately rendering the kidneys dysfunctional, and the condition potentially progresses to end-stage renal disease. , $^{, 9,11,12}$ 
Autosomal dominant PKD (ADPKD) is the most common genetic form of PKD, occurring in 1 in 400 to 1 in 1,000 live births and it affects $\sim 500,000$ Americans. ${ }^{1,5,9,12}$ ADPKD may be asymptomatic for several decades, but as cysts increase in size and number, symptoms and serious sequelae develop. Autosomal recessive PKD is much rarer than ADPKD and it occurs in only about 1 in 20,000 live births. ${ }^{1,5}$ Symptom presentation is more rapid and occurs at an earlier age for persons with autosomal recessive PKD than for persons with ADPKD, so ADPKD is more likely to be a long-term chronic condition. ${ }^{1,12,13}$

Although there are few reports on the economic burden associated with ADPKD, literature reports indicate that chronic kidney disease in general is a source of substantial clinical and economic burden. Smith et al reported that among enrollees of a large health maintenance organization, health care expenditures for chronic kidney disease patients were $\sim$ US\$8,000 more than those without chronic kidney disease. ${ }^{14}$ Baumeister et al, in a study of a general population, reported that persons with chronic kidney disease had $\sim 68 \%$ higher total costs, $60 \%$ higher inpatient costs, $48 \%$ higher drug costs, and a $1.40 \%$ higher relative risk of hospitalization compared to persons without chronic kidney disease. ${ }^{15} \mathrm{PKD}$, in particular, has been identified as the leading hereditary cause of end-stage renal disease in the United States. ${ }^{16}$ Renal failure occurs by age 60 years in $\sim 50 \%$ of patients with ADPKD, requiring dialysis or kidney transplant. ${ }^{5}$ Lentine et al estimated all-cause health care costs for persons with $\mathrm{ADPKD}^{17}$ and reported mean annual unadjusted all-cause total health care expenditures ranging from US\$26,521 at baseline to US\$140,139 at the advanced kidney disease level. ${ }^{17}$

There is limited evidence describing incremental economic burden of ADPKD. Blanchette et al reported higher all-cause hospital-based inpatient resource utilization among ADPKD patients compared to persons with chronic kidney disease. ${ }^{18}$ Knight et al reported that health care resource use and expenditures over a 6-month duration were greater among ADPKD patients compared to non-ADPKD controls matched on age and gender. ${ }^{19}$ However, the study did not match on or adjust for comorbidities. Additional evidence regarding the incremental burden of ADPKD may offer valuable input in understanding the societal impact of the disease. The specific study objectives were to:

1. Determine incremental health care resource utilization associated with ADPKD by categories including hospitalizations, hospital days, nursing home confinements, nursing home days, outpatient visits, and emergency room visits, and
2. Determine incremental health care expenditures associated with ADPKD by categories including hospital expenditures, nursing home expenditures, outpatient expenditures, emergency room expenditures, medication expenditures, and total health care expenditures.

\section{Methods}

\section{Study design}

An observational database analysis was conducted using information from a large administrative database. Individuals aged 18 years or older with ADPKD were identified using International Classification of Disease, Ninth Revision, Clinical Modification (ICD-9-CM) codes. A 1-year study interval from April 1, 2011 through March 31, 2012 was used in the analysis.

\section{Data source}

Health insurance claims data from the OptumInsight ${ }^{\mathrm{TM}} \mathrm{Clin}$ formatics ${ }^{\mathrm{TM}}$ DataMart database (Optum, Eden Prairie, MN, USA) between April 1, 2011 and March 31, 2012 were used to conduct an observational database analysis. The Clinformatics ${ }^{\mathrm{TM}}$ DataMart database includes medical and pharmacy claims for 13 million privately insured lives covering all census regions of the United States. Data elements used in this study included enrollment records, patient demographics, inpatient and outpatient medical services, and prescription drug dispensing records. The database contained only deidentified information. To account for pricing variation across providers or geographic regions, prices in the database are standardized to reflect allowed payments from a single source with a consistent payment plan. The standardized prices have been routinely used in the analysis of expenditures in prior literature..$^{20,21}$

\section{Study participants}

Persons 18 years or older, enrolled in a tracked health plan during the 12-month period from April 1, 2011 through March 31, 2012, and having a diagnosis for "polycystic kidney, autosomal dominant" (753.13) or for "polycystic kidney, unspecified type" (753.12) were selected for inclusion in the sample. Persons having ICD-9-CM diagnosis codes for "polycystic kidney, autosomal recessive" (753.14) or "cystic kidney disease" (753.1) were excluded from the ADPKD group because of their differing clinical presentation and prognosis. Each individual in the ADPKD group was linked based on age and gender with an individual without ADPKD. The non-ADPKD group consisted of individuals who satisfied all inclusion criteria for the ADPKD group but did not have a diagnosis for ADPKD. Exclusion criteria for the non-ADPKD 
group were a diagnosis for autosomal recessive PKD (753.14), cystic kidney disease (753.1), chronic kidney disease stage 3 to stage $6(585.3,585.4,585.5,585.6,585.9,586)$, nephrotic syndrome $(581,581.0,581.1,581.2,581.3,581.8,581.9$, 581.81, 583.81, V13.03), diabetic kidney disease (250.4, $250.40,250.41,250.42,250.43,249.4,249.40,249.41)$, or kidney stones associated with cystic kidney disease (diagnosis code: 274.11, procedure codes: 55.03, 55.04, 59.95, 56.0, $98.5,98.51)$. Renal diseases were excluded from the control sample so as to avoid potential confounding from associated resource utilization and expenditures from those renal diseases. For sub-group analysis, ADPKD cases with end-stage renal disease were identified by presence of ICD-9-CM code for end-stage renal disease (585.6), current procedural terminology codes for chronic hemodialysis for ages 20 years and over (90960, 90961, 90963), HCPCS codes used for center based patients on dialysis (G0308-G0319), HCPCS codes used for home dialysis patients (G0320-G0323), ICD-9 code for renal transplant (v42.0), and CPT code for renal transplant (50360, 50365). This study was approved by the Institutional Review Board of Purdue University.

\section{Quantitative variables and covariates}

Health care resource utilization by specific category was calculated for each individual and health care expenditures by specific categories were computed by adding standard prices from insurance claims. All costs were adjusted to 2018 US dollars. Demographic variables including year of birth, gender, and geographical region were extracted from eligibility and claims files. A modified Charlson Comorbidity Index developed by Romano et al was constructed for the sample, in which renal diseases were excluded in the computation of comorbidity index scores. ${ }^{22}$

\section{Statistical methods}

Data were analyzed using SAS for Unix Version 9.2 and STATA for Unix version 12. Frequency distributions were developed to describe the sample, and chi-square tests were used to assess statistical differences between persons with or without ADPKD based on demographic variables and clinical variables. Unadjusted means and 95\% CIs, and medians for utilization and expenditure in each category were also computed and Wilcoxon-Mann-Whitney test was used to detect differences between the groups.

Individual zero-inflated negative binomial regression models were developed to estimate independent association between ADPKD and utilization of health services, including hospitalizations, hospital days, nursing home confinements, nursing home days, outpatient visits, and emergency room visits. A binary predictor variable for ADPKD was included in each model, and covariates in each model included age, gender, comorbidity index score, cardiovascular disease, diabetes, and geographical region, to adjust for these risk factors. Similarly, individual zero-inflated negative binomial regression models were also developed for health care expenditures including total expenditures, hospital expenditures, nursing home expenditures, outpatient expenditures, emergency room expenditures, and medication expenditures, with the same predictor variable and covariates described earlier. Subgroup analysis examined incremental resource utilization and expenditures associated with ADPKD across sub-groups with ADPKD and end-stage renal disease and those with ADPKD but without end-stage renal disease. ADPKD cases with a diagnosis of end-stage renal disease and their comparison individuals were one sub-group and cases without end-stage renal disease and their comparison individuals formed the other sub-group. Individual zero-inflated negative binomial regression models, as described for the overall analysis, were also developed to estimate incremental resource utilization and expenditures by category for each sub-group, adjusting for risk factors.

\section{Results \\ Sample characteristics}

Out of 10,500,664 persons at least 18 years old and enrolled during the 12-month period from April 1, 2011 through March 31, 2012, there were 4,020 individuals having a diagnosis for ADPKD. After excluding 115 individuals who either had a diagnosis of autosomal recessive PKD or unspecified cystic kidney disease and 61 persons who had no medical claim information, a total of 3,844 individuals remained in the ADPKD group. The individuals with an ADPKD diagnosis were linked one-to-one with individuals without ADPKD. As shown in Table 1, the sample was 53\% female, with a mean age \pm SD of $49.15 \pm 13.67$ years. Approximately, 55\% were between 45 and 64 years old. As also shown in Table 1, a significantly higher proportion of individuals in the ADPKD group had Charlson Comorbidity Index scores greater than zero $(55.8 \%$ vs $37.5 \%, P<0.05)$. The cohort with ADPKD also had proportionally higher diagnosis of cardiovascular disease $(25.6 \%$ vs $13.3 \%, P<0.05)$ and diabetes $(14.1 \%$ vs $10.0 \%, P<0.05)$. Since hypertension is a major cardiovascular risk factor among persons with ADPKD, it was not surprising to observe a higher incidence of cardiovascular disease among the ADPKD cohort. Multivariate regression models estimating incremental resource use and expenditures 
Table I Distribution of total study sample and distribution of persons with or without ADPKD by Charlson Comorbidity Index, presence of cardiovascular disease, presence of diabetes, and geographical region

\begin{tabular}{|c|c|c|c|c|}
\hline \multirow[t]{2}{*}{ Characteristic } & \multirow{2}{*}{$\begin{array}{l}\text { Total sample } \\
(n=7,688) \\
\text { Number }(\%)\end{array}$} & \multirow{2}{*}{$\begin{array}{l}\text { With ADPKD } \\
(n=3,844) \\
\text { Number }(\%)\end{array}$} & \multirow{2}{*}{$\begin{array}{l}\text { Without ADPKD } \\
(n=3,844) \\
\text { Number }(\%)\end{array}$} & \multirow[t]{2}{*}{$P$-value ${ }^{a}$} \\
\hline & & & & \\
\hline \multicolumn{5}{|l|}{ Age (years) } \\
\hline Under 35 & $1,242(16.2)$ & $621(16.2)$ & $621(16.2)$ & ND \\
\hline $35-44$ & $1,496(19.5)$ & $748(19.5)$ & $748(19.5)$ & ND \\
\hline $45-54$ & $2,086(27.1)$ & $1,043(27.1)$ & $1,043(27.1)$ & ND \\
\hline $55-64$ & $2,118(27.5)$ & $1,059(27.5)$ & $1,059(27.5)$ & ND \\
\hline 65 and over & $746(9.7)$ & $373(9.7)$ & $373(9.7)$ & ND \\
\hline \multicolumn{5}{|l|}{ Gender } \\
\hline Female & $4,094(53.2)$ & $2,047(53.2)$ & $2,047(53.2)$ & ND \\
\hline Male & $3,594(46.8)$ & I,797 (46.8) & I,797 (46.8) & ND \\
\hline \multicolumn{5}{|c|}{ Charlson Comorbidity Index } \\
\hline 0 & $4,103(53.4)$ & $\mathrm{I}, 700(44.2)$ & $2,403(62.5)$ & $<0.001$ \\
\hline 1 & I,447 (I8.8) & $781(20.3)$ & $666(17.3)$ & 0.003 \\
\hline 2 & $823(10.7)$ & $464(12.1)$ & $359(9.3)$ & $<0.001$ \\
\hline 3 & $491(6.4)$ & $312(8.1)$ & $179(4.7)$ & $<0.001$ \\
\hline 4 & $280(3.6)$ & $192(5.0)$ & $88(2.3)$ & $<0.001$ \\
\hline 5 & $169(2.2)$ & $120(3.1)$ & $49(1.3)$ & $<0.001$ \\
\hline 6 or more & $375(4.9)$ & $275(7.2)$ & $100(2.6)$ & $<0.001$ \\
\hline Cardiovascular disease & I,493 (19.4) & $983(25.6)$ & $510(13.3)$ & $<0.001$ \\
\hline Diabetes & $926(12.0)$ & $542(14.1)$ & $384(10.0)$ & $<0.001$ \\
\hline \multicolumn{5}{|l|}{ Region } \\
\hline Northeast & $801(10.4)$ & $419(10.9)$ & $382(10.0)$ & 0.2 \\
\hline Midwest & $2,101(27.2)$ & $\mathrm{I}, 008(26.2)$ & I,093 (28.4) & 0.06 \\
\hline South & $3,455(44.9)$ & I,777 (46.2) & $\mathrm{I}, 678(43.7)$ & 0.09 \\
\hline West & $1,327(17.4)$ & $640(16.7)$ & $687(17.9)$ & 0.2 \\
\hline Missing & $4(0.1)$ & $0(0.0)$ & $4(0.0)$ & NA \\
\hline
\end{tabular}

Notes: Persons with ADPKD were matched I:I with persons without ADPKD; abased on chi-squared tests.

Abbreviations: ND, no difference; NA, not available; ADPKD, autosomal dominant polycystic kidney disease.

associated with ADPKD were adjusted for each of the clinical and demographic variables.

\section{Unadjusted annual health care resource utilization and expenditures among persons with or without ADPKD Unadjusted resource utilization}

Table 2 shows mean and median resource use by category for the group with ADPKD and the group without ADPKD. Mean annual unadjusted hospitalizations were three times higher among individuals with ADPKD (0.24) as compared to those without ADPKD (0.085), $P<0.001$. Individuals with ADPKD had four times greater mean annual unadjusted hospital days (2.0) as compared to individuals without ADPKD (0.45), $P<0.001$. Mean annual unadjusted outpatient visits were more than twice as great among individuals with ADPKD (21.1) as compared to those without ADPKD (9.7), $P<0.001$.

\section{Unadjusted expenditures}

Mean unadjusted annual health care expenditures are reported in Table 2. Mean annual unadjusted hospital expenditures were more than four times higher among individuals with ADPKD (US\$7,252) as compared to individuals without ADPKD (US\$1,619), $P<0.001$. Mean annual unadjusted expenditures from nursing homes were also sixfold higher for the cohort with ADPKD (US\$275) as compared to the cohort without ADPKD (US\$45), $P<0.001$. Unadjusted mean annual outpatient expenditures were almost four times higher among individuals with ADPKD (US\$13,777) than for individuals without ADPKD (US\$3,519), $P<0.001$. Mean annual unadjusted medication expenditures for individuals with ADPKD (US\$3,860) were $\sim 2.5$ times higher when compared to individuals without ADPKD (US\$1,506), $P<0.001$. Mean annual unadjusted total health care expenditures among individuals with ADPKD (US\$25,363) were more than three and one-half times as high as for those individuals without ADPKD (US\$6,799), $P<0.001$. 
Table 2 Unadjusted annual health care resource utilization and expenditures among persons with and without ADPKD

\begin{tabular}{|c|c|c|c|c|c|}
\hline \multirow[t]{2}{*}{ Category } & \multicolumn{2}{|c|}{ Individuals with APKD $(n=3,844)$} & \multicolumn{2}{|c|}{ Individuals without ADPKD $(n=3,844)$} & \multirow[t]{2}{*}{$P$-value ${ }^{a}$} \\
\hline & Mean $(95 \% \mathrm{Cl})$ & Median & Mean $(95 \% \mathrm{Cl})$ & Median & \\
\hline \multicolumn{6}{|l|}{ Health resource utilization } \\
\hline Hospitalizations & $0.24(0.21-0.26)$ & 0 & $0.085(0.074-0.096)$ & 0 & $<0.001$ \\
\hline Hospital days & $2.0(1.6-2.5)$ & 0 & $0.45(0.27-0.62)$ & 0 & $<0.001$ \\
\hline Nursing home confinements & $0.011(0.0077-0.015)$ & 0 & $0.0031(0.0013-0.0048)$ & 0 & $<0.001$ \\
\hline Nursing home days & $0.33(0.18-0.49)$ & 0 & $0.07 I(0.02 I-0.12)$ & 0 & $<0.001$ \\
\hline Outpatient visits & $21.1(20.3-21.9)$ & 13 & $9.7(9.3-10.0)$ & 6 & $<0.001$ \\
\hline Emergency room visits & $1.0(0.96-1.2)$ & 0 & $0.68(0.6 \mathrm{I}-0.76)$ & 0 & $<0.001$ \\
\hline \multicolumn{6}{|l|}{ Health care expenditures } \\
\hline Hospital expenditures & $7,252(6,204-8,302)$ & 0 & $1,619(1,347-1,892)$ & 0 & $<0.001$ \\
\hline Nursing home expenditures & $275(143-407)$ & 0 & $45(14-75)$ & 0 & $<0.001$ \\
\hline Outpatient expenditures & $\mid 3,777(|2,604-| 4,95 \mid)$ & 3,238 & $3,519(3,052-3,986)$ & $\mathrm{I}, 048$ & $<0.001$ \\
\hline Emergency room expenditures & $196(177-215)$ & 0 & $109(97-121)$ & 0 & $<0.001$ \\
\hline Medication expenditures & $3,860(3,627-4,093)$ & 1,088 & $1,506(I, 347-I, 664)$ & 252 & $<0.001$ \\
\hline Total health care expenditures & $25,363(223,560-27,167)$ & 6,639 & $6,799(6,|85-7,4| I)$ & 2,049 & $<0.001$ \\
\hline
\end{tabular}

Notes: aUnadjusted mean health care resource utilization and unadjusted mean health care expenditures between individuals with and without ADPKD were compared using the Wilcoxon-Mann-Whitney test.

Abbreviation: ADPKD, autosomal dominant polycystic kidney disease.

\section{Incremental annual health care resource utilization and expenditures associated with ADPKD}

Data distributional characteristics were examined, and sequential assessment of potential regression techniques was conducted to select an appropriate model, for analysis of incremental resource utilization, and expenditures associated with ADPKD. Residuals of ordinary least squares regression models with both untransformed and logtransformed utilization and expenditure variables showed a violation of assumptions of normality and constant variance. $P$-values $<0.05$ for each Kolmogorov-Smirnov test showed that residuals for each ordinary least squares regression model were not normally distributed. ${ }^{23}$ Results of each Glejser test showed $P$-values $<0.05$, confirming that residuals were significantly associated with independent variables. ${ }^{24}$ Therefore, generalized linear models were developed and tested for model fit. Medical resource utilization and expenditure variables had a high number of observations with zeros. For example, out of 7,688 cases and controls, 6,804 had zero inpatient hospitalizations, 6,805 had zero inpatient hospital expenditures, 569 had zero outpatient visits, 572 had zero outpatient expenditures, 5,460 had zero emergency room visits, and 1,127 had zero medication expenditures. Overdispersion of dependent variables evidenced by $P$-values $<0.05$ for Vuong tests suggested a need for a zero-inflated generalized linear model. ${ }^{25,26} \mathrm{Sig}$ nificant likelihood ratio tests, shown by $P$-values $<0.05$, indicated that for each dependent variable, zero-inflated negative binomial models were more suitable than zeroinflated Poisson models. ${ }^{26-29}$

\section{Incremental resource utilization}

Results from multivariate zero-inflated negative binomial regression models for each health care resource utilization type are reported in Table 3. For nursing home confinements, results from regression models were not available since the number of nursing home confinements were so low that models did not converge. ADPKD was associated with many more outpatient visits as reflected in a mean (SD) of $6.9(0.28)(P<0.001)$ incremental outpatient visits. Although ADPKD was associated with incrementally more hospitalizations (0.087 [0.011], $P<0.05)$, the impact of ADPKD on hospital resource utilization may be better reflected in the mean incremental hospital days (0.68 [0.090], $P<0.001)$. Emergency room visits were also incrementally greater among persons with ADPKD ( 0.29 [0.055], $P<0.001)$.

\section{Incremental expenditures}

As shown in Table 3, the mean incremental total health care expenditure associated with ADPKD was US $\$ 8,639$ (\$431) $(P<0.001)$. The mean incremental expenditures for outpatient expenditures associated with ADPKD (US\$4,918 [\$181], $P<0.001)$ accounted for the largest incremental expenditure. Hospital expenditures had the second largest incremental expenditure associated with ADPKD (US\$2,603 [\$263], $P<0.001)$. Incremental expenditures associated with 


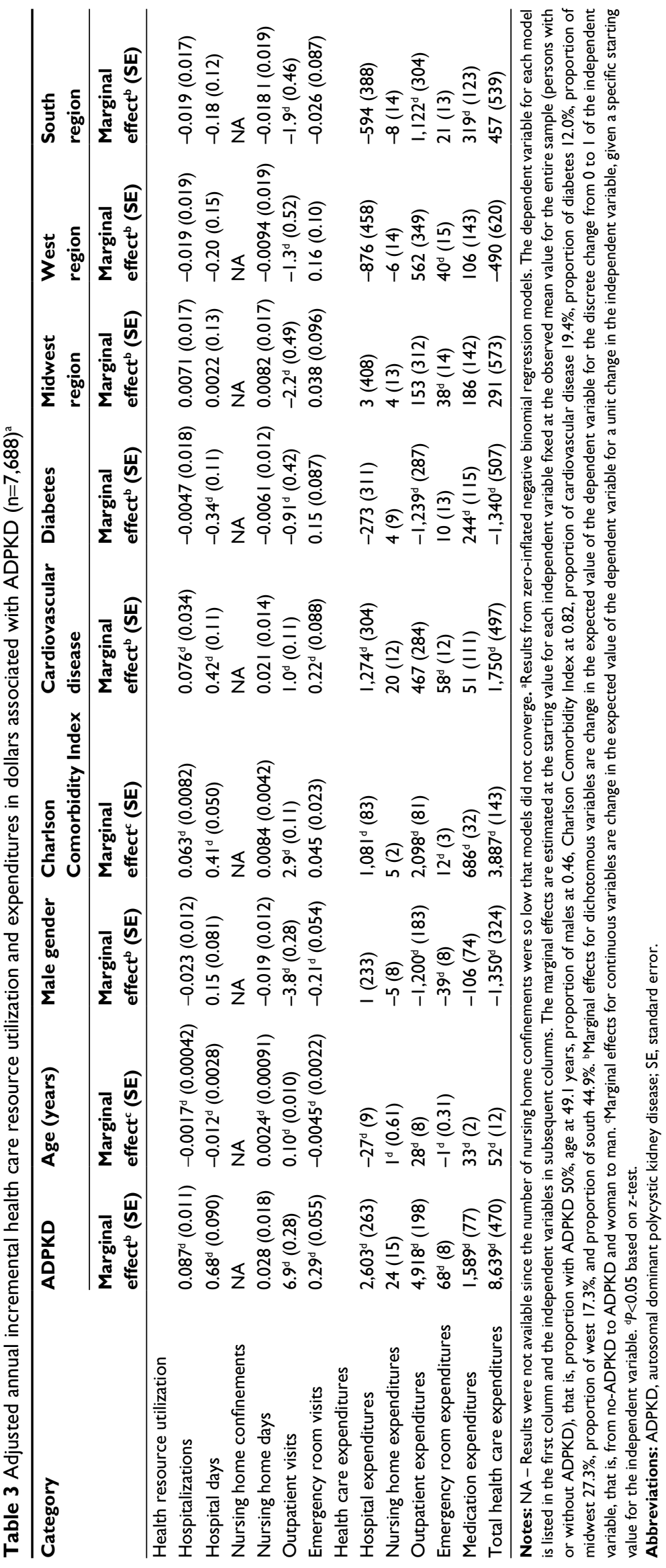


ADPKD for medication expenditures were US\$1,589 (\$77) $(P<0.001)$ and emergency room expenditures were US\$68 $(\$ 8)(P<0.001)$.

\section{Sub-group analysis by whether diagnosed with end-stage renal disease}

Among 3,844 persons with ADPKD, 802 were diagnosed with end-stage renal disease and 3,042 persons with ADPKD had not been diagnosed with end-stage renal disease. Table 4 shows incremental health care resource utilization associated with ADPKD, based on results of zero-inflated negative binomial models by sub-group. Significant incremental mean (standard error) hospitalizations (0.29 [0.042], $P<0.001)$ and hospital days $(1.8[0.38], P<0.001)$ were associated with ADPKD in the sub-group with end-stage renal disease. There were also significant incremental hospitalizations (0.061 [0.019], $P<0.001)$ and hospital days $(0.47$ [0.081], $P<0.001)$ associated with ADPKD in the sub-group without end-stage renal disease. Although the magnitude of estimated incremental hospitalizations and hospital days were greater in the sub-group with end-stage renal disease as compared to estimates from the model including all persons regardless of end-stage renal disease status, ADPKD was still associated with significant incremental hospitalizations and hospital days in the sub-group containing only persons without end-stage renal disease. The magnitude of the estimates of incremental hospitalizations in the group without end-stage renal disease (0.061 [0.019], $P<0.001$ ) were similar to those from the model including everyone regardless of end-stage renal disease status $(0.087$ [0.011], $P<0.001)$. The same was true for the magnitude of incremental hospital days estimated among those without end-stage renal disease (0.47 [0.081], $P<0.001)$, as compared to that from the model for all individuals regardless of end-stage renal disease status $(0.68$ [0.090], $P<0.001)$. Similarly, although the sub-group with end-stage renal disease (20.7 [1.0], $P<0.001)$ had several times higher incremental outpatient visits associated with ADPKD as compared to the estimates for all individuals with ADPKD regardless of end-stage renal disease status (6.9 [0.28], $P<0.001)$, the ADPKD sub-group without end-stage renal disease (4.1 [0.36], $P<0.001)$ had mean incremental outpatient visits close in magnitude to those from the inclusive sample. In contrast, the sub-group analysis revealed that there were not significant incremental emergency room visits associated with ADPKD in the sub-group with end-stage renal disease, but that the sub-group without end-stage renal disease did have significant incremental emergency room visits associated with ADPKD $(0.34[0.060], P<0.001)$, and of similar magnitude to that from the model for all individuals regardless of end-stage renal disease status $(0.29$ [0.055], $P<0.001)$.

Results from sub-group analyses of incremental expenditures associated with ADPKD are also reported in Table 4. The ADPKD sub-group with end-stage renal disease had mean incremental total expenditures associated with ADPKD of US\$38,962 $(\$ 6,181)(P<0.001)$, which were much higher than those for the sample including all persons with ADPKD regardless of end-stage renal disease status (US\$8,639 [ $\$ 470], P<0.001)$. Although the sub-group without end-stage renal disease had lower incremental mean total expenditures (US\$2,944 [\$417], $P<0.001$ ) associated with ADPKD than for the group including everyone regardless of end-stage renal disease status, the incremental expenditures were still large. Similar relationships were seen in comparison across the sub-group with end-stage renal disease, the sub-group without end-stage renal disease, and the overall sample, for incremental hospital expenditure of US\$8,069 $(\$ 1,079)$ $(P<0.001)$, US\$1,507 (\$227) $(P<0.001)$, and US $\$ 2,603$ (\$263) $(P<0.001)$; outpatient expenditures of US\$23,403 $(\$ 1,174)(P<0.001), \mathrm{US} \$ 1,276(\$ 131)(P<0.001)$, and US\$4,918 (\$198) $(P<0.001)$; and medication expenditures of US\$6,743 (\$336) $(P<0.001)$, US\$541 (\$60) $(P<0.001)$, and US $\$ 1,589$ ( $\$ 77), P<0.001$, respectively. However, incremental emergency room expenditures associated with ADPKD were of similar magnitude for the sub-group with end-stage renal disease (US\$77 (\$21), $P<0.001$ ), the sub-group without end-stage renal disease ( $\$ 67$ [ $\$ 9$ ], $P<0.001$ ), and the overall group incorporating all individuals regardless of end-stage renal disease status $(\$ 68[\$ 8], P<0.001)$.

\section{Discussion}

Administrative insurance records were examined to assemble a cohort of patients with ADPKD and to estimate all-cause health care resource utilization and health care expenditures for those patients. Incremental health care resource utilization and incremental health care expenditures associated with ADPKD were also estimated. To account for pricing variation across providers or geographic regions, standardized prices of health care expenditures were used in this study.

A diagnosis of ADPKD was associated with substantial incremental resource use and expenditures, even after adjusting for risk factors. Incremental resource utilization (standard error) from outpatient visits was large (6.9 [0.28]). Significant incremental resource utilization associated with ADPKD for hospitalizations (0.087 [0.011]), hospital days $(0.68$ [0.090]), and emergency room visits (0.29 [0.055]) 


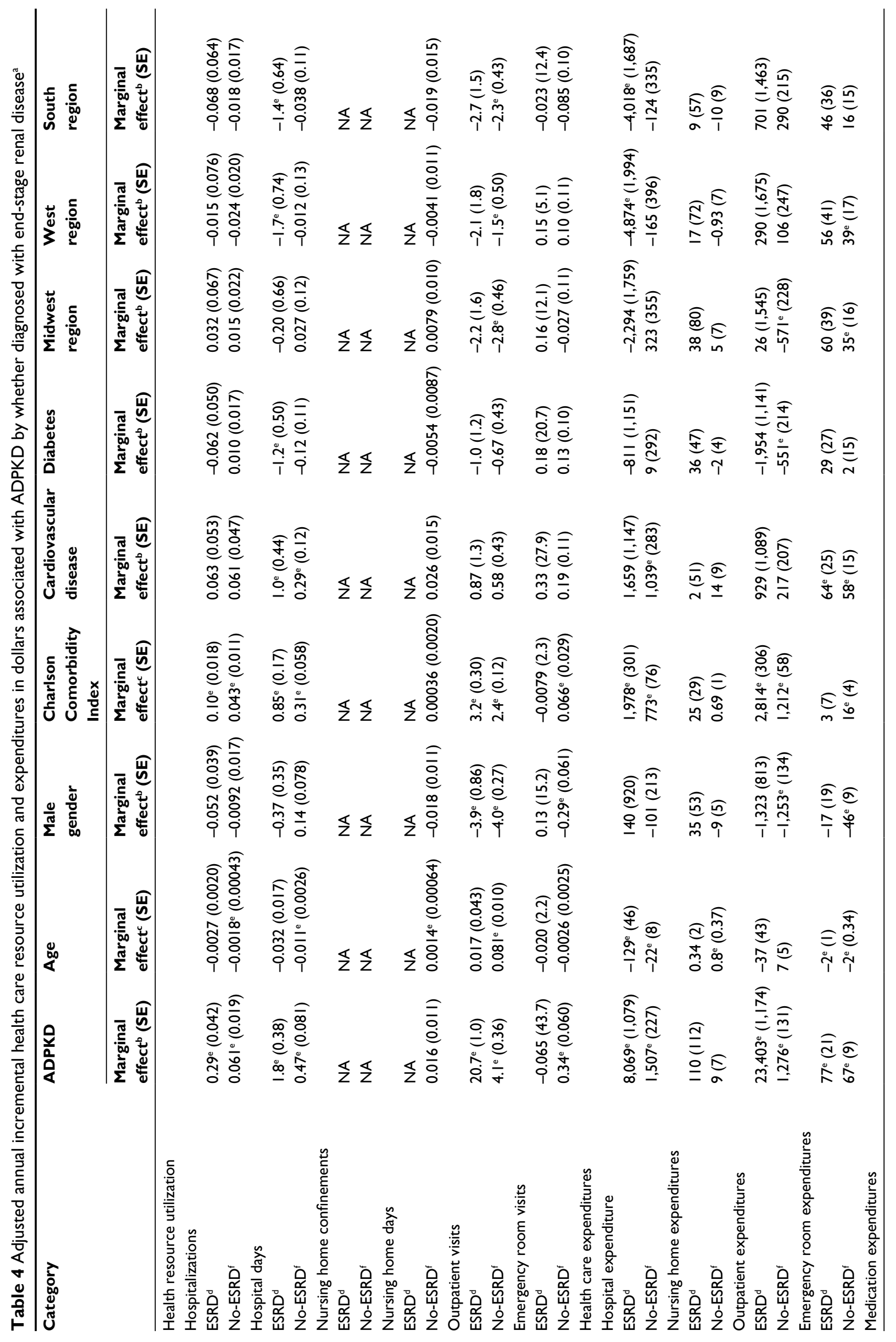




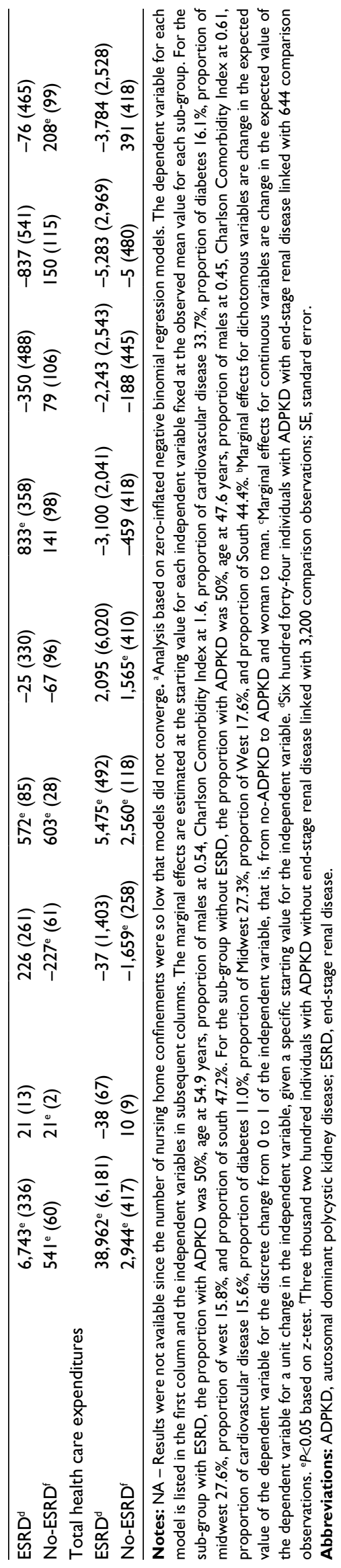

was also found. Considerable incremental expenditures associated with ADPKD from hospital expenditures $(\$ 2,603$ [\$263]), incremental medication expenditures (\$1,589 [\$77]), and substantially high incremental outpatient expenditures (\$4,918 [\$198]) were also observed.

Alexander et al reported that chronic kidney disease was an independent predictor of health care resource utilization after accounting for other risk factors, consistent with findings from this study. ${ }^{28}$ Mean annual all-cause total health care expenditures reported by Lentine et al were US $\$ 26,521$ at early stages of renal function decline among individuals with ADPKD and rose to US\$140,139 when patients reached end-stage renal disease. ${ }^{17}$ As reported by Knight et al, mean unadjusted hospitalization costs were $2-8$ times higher and mean unadjusted hospital outpatient visits were $\sim 3-4$ times higher for ADPKD patients at earlier stages of chronic kidney disease compared to age- and gender-matched controls (who had no ADPKD). ${ }^{19}$ However, Blanchette et al reported a 44\% shorter average length of hospital stay for the ADPKD cohort as compared with persons with chronic kidney disease. ${ }^{18}$ This present study reports that incremental total health care expenditures associated with ADPKD were higher for the ADPKD sub-group with end-stage renal disease (US\$38,962 $[\$ 6,181]$ ) than for the ADPKD sub-group without end-stage renal disease (US\$2,944 [\$417]), consistent with trends in all-cause expenditures by kidney function levels as reported by Lentine et al and hospitalization costs and hospital outpatient visits at earlier stages of chronic kidney disease as reported by Knight et al. ${ }^{17,19}$

Although individuals with ADPKD and end-stage renal disease had the highest incremental expenditures, a diagnosis of ADPKD without end-stage renal disease was associated with considerable incremental inpatient expenditures, outpatient expenditures, medication expenditures, and total health care expenditures. These expenditures could be explained - at least in part - by both acute and chronic burdens of ADPKD. Acutely, ADPKD patients may experience episodes of painful renal cyst rupture, hematuria, nephrolithiasis, urinary tract infections, and rare intracranial aneurysms. Chronically, ADPKD patients have high rates of hypertension, costs associated with screening imaging of the kidneys and brain, and management of the sequelae of chronic kidney disease and its associated cardiovascular disease. ${ }^{5}$

\section{Study limitations}

At the time the study was conducted, most recent data available was up until March 31, 2012. Since the time that this study was conducted, there has been only one treatment (tolvaptan) that 
was approved for use in this patient population and has been available for patients since April $2018 .{ }^{29}$ Since this treatment only recently entered the US market, there is currently limited data on the average additional cost per ADPKD patient at the time of the writing of this manuscript. However, given the relatively high cost of tolvaptan treatment, it is possible that the incremental health care expenditures will grow in future years. The database used in this study includes medical and pharmacy claims for 13 million privately insured lives in the United States which limits the generalizability of study findings. Another limitation of this study was that ICD-9-CM codes were used to identify individuals with ADPKD. Also, the comorbidities used for the Charlson Comorbidity Index were identified using ICD-9-CM codes. Use of diagnostic codes in claims to identify diagnosis is known to be imperfect due to variations in coding. ${ }^{30}$ However, the set of codes used in this study to identify ADPKD have been utilized in prior studies. ${ }^{17,31}$ Since the data source included individuals with private insurance, it is possible that some of the health care costs in the end-stage renal disease population were underestimated in this study, as the Centers for Medicare and Medicaid Services begins to pay for a portion of the medical care once the diagnosis of end-stage renal disease is made.

\section{Conclusion}

In conclusion, ADPKD was associated with significant incremental health care resource utilization and expenditures even after adjusting for age, gender, comorbidities, cardiovascular disease, diabetes, and geographical region. Considerable illness burden was found even before patients reached endstage renal disease.

\section{Acknowledgments}

Funding for this research was provided by Pfizer, Inc., New York, NY. Work for this research was performed at Purdue University, West Lafayette, Indiana. Portions of this data were presented previously at the 18th Annual Meeting of the International Society for Pharmacoeconomics and Outcomes Research (ISPOR), May 18-22, 2013, New Orleans, LA, and the 19th Annual Meeting of the International Society for Pharmacoeconomics and Outcomes Research (ISPOR), May 31-June 04, 2014, Montreal, QC, Canada.

\section{Disclosure}

At the time of this research, Neeraj $\mathrm{N}$ Iyer was an employee of Purdue University, and currently is an employee of Novo Nordisk Inc. Joseph Thomas III is an employee of Purdue University. Nicholas J Vendetti, Daniel I Levy, and Jack
Mardekian are employees of Pfizer, Inc. Marko A Mychaskiw was an employee of Pfizer, Inc. at the time that the research was conducted and the manuscript initiated, and currently is an employee of Eli Lilly and Company. Joseph Thomas III and Neeraj N Iyer were paid consultants to Pfizer, Inc. in connection with the development of this manuscript. The authors report no other conflicts of interest in this work.

\section{References}

1. Pirson Y, Chauveau D. Cystic disease of the kidney. In: Glassock RJ, Cohen AH, Grünfeld JP, editors. Atlas of Diseases of the Kidney. Vol 2. Wiley-Blackwell; Hoboken, NJ. 1999.

2. Alehan FK, Gürakan B, Ağildere M. Familial arachnoid cysts in association with autosomal dominant polycystic kidney disease. Pediatrics. 2002;110(1 Pt 1):e13.

3. Alpern MB, Dorfman RE, Gross BH, Gottlieb CA, Sandler MA. Seminal vesicle cysts: association with adult polycystic kidney disease. Radiology. 1991;180(1):79-80.

4. Danaci M, Akpolat T, Baştemir M, et al. The prevalence of seminal vesicle cysts in autosomal dominant polycystic kidney disease. Nephrol Dial Transplant. 1998;13(11):2825-2828.

5. Grantham JJ. Clinical practice. Autosomal dominant polycystic kidney disease. N Engl J Med. 2008;359(14):1477-1485.

6. Nicolau C, Torra R, Bianchi L, et al. Abdominal sonographic study of autosomal dominant polycystic kidney disease. J Clin Ultrasound. 2000;28(6):277-282.

7. Pirson Y, Chauveau D, Torres V. Management of cerebral aneurysms in autosomal dominant polycystic kidney disease. J Am Soc Nephrol. 2002;13(1):269-276.

8. Schievink WI, Huston J, Torres VE, Marsh WR. Intracranial cysts in autosomal dominant polycystic kidney disease. J Neurosurg. 1995;83(6):1004-1007.

9. Torres VE, Harris PC, Pirson Y. Autosomal dominant polycystic kidney disease. Lancet. 2007;369(9569):1287-1301.

10. Wijdicks EF, Torres VE, Schievink WI. Chronic subdural hematoma in autosomal dominant polycystic kidney disease. Am J Kidney Dis. 2000;35(1):40-43.

11. Torres VE, Grantham JJ. Cystic diseases of the kidney. In: Brenner BM, editor. Brenner and Rector's the Kidney. 8th ed. Philadelphia, PA: Saunders Elsevier; 2007.

12. Wilson PD. Polycystic kidney disease. N Engl J Med. 2004;350(2): 151-164.

13. Dell KMR, Avner ED. Polycystic kidney disease, autosomal recessive. In: Pagon RA, Adam MP, Bird TD, editors. GeneReviews ${ }^{\mathrm{TM}}$ [Internet]. 2001. Available from: http://www.ncbi.nlm.nih.gov/books/NBK1326/. Accessed August 20, 2018.

14. Smith DH, Gullion CM, Nichols G, Keith DS, Brown JB. Cost of medical care for chronic kidney disease and comorbidity among enrollees in a large HMO population. J Am Soc Nephrol. 2004;15(5):1300-1306.

15. Baumeister SE, Böger CA, Krämer BK, et al. Effect of chronic kidney disease and comorbid conditions on health care costs: a 10-year observational study in a general population. Am J Nephrol. 2010;31(3):222-229.

16. United States Renal Data System. USRDS 2011 Annual Data Report: ESRD Reference Tables. Bethesda: NIH, National Institute of Diabetes and Digestive and Kidney Diseases; 2011.

17. Lentine KL, Xiao H, Machnicki G, Gheorghian A, Schnitzler MA. Renal function and healthcare costs in patients with polycystic kidney disease. Clin J Am Soc Nephrol. 2010;5(8):1471-1479.

18. Blanchette CM, Craver C, Belk KW, et al. Hospital-based inpatient resource utilization associated with autosomal dominant polycystic kidney disease in the US. J Med Econ. 2015;18(4):303-311.

19. Knight T, Schaefer C, Krasa H, et al. Medical resource utilization and costs associated with autosomal dominant polycystic kidney disease in the USA: a retrospective matched cohort analysis of private insurer data. Clinicoecon Outcomes Res. 2015;7:123. 
20. Kimball AB, Guérin A, Tsaneva M, et al. Economic burden of comorbidities in patients with psoriasis is substantial. J Eur Acad Dermatol Venereol. 2011;25(2):157-163.

21. Vekeman F, Lamori JC, Laliberté F, et al. Risks and cost burden of venous thromboembolism and bleeding for patients undergoing total hip or knee replacement in a managed-care population. J Med Econ. 2011;14(3):324-334.

22. Romano PS, Roos LL, Jollis JG. Adapting a clinical comorbidity index for use with ICD-9-CM administrative data: differing perspectives. J Clin Epidemiol. 1993;46(10):1075-1079.

23. D'Agostino RB, Stephens MA. Goodness-of-Fit Techniques. New York: Marcel Dekker; 1986.

24. Glejser H. A new test for heteroskedasticity. J Am Stat Assoc. 1969;64(325):316-323.

25. Long SJ, Freese J. Regression Models for Categorical Dependent Variables Using Stata. 2nd ed. Thousand Oaks, CA: Stata Press; 2006.

26. Vuong QH. Likelihood ratio tests for model selection and non-nested hypotheses. Econometrica. 1989;57(2):307-333.
27. Cameron AC, Trivedi PK. Econometric models based on count data Comparisons and applications of some estimators and tests. $\mathrm{J} \mathrm{Appl}$ Econ. 1986;1(1):29-53.

28. Alexander M, Bradbury BD, Kewalramani R, Barlev A, Mohanty SA, Globe D. Chronic kidney disease and US healthcare resource utilization in a nationally representative sample. Am J Nephrol. 2009;29(5): 473-482.

29. Kluwer W. Medi-Span Price Rx. Available from: https://www.wolterskluwercdi.com/price-rx/. Accessed June 20, 2018.

30. Romano PS, Mark DH. Bias in the coding of hospital discharge data and its implications for quality assessment. Med Care. 1994;32(1):81-90.

31. Perrone RD, Kausz AT, Chavers BM, Weinhandl ED, Collins AJ. Incidence of Autosomal Dominant Polycstic Kidney Disease (ADPKD) Among ESRD Patients in the United States, 1991-2003. United States Renal Data System; 2005. Available from: https://www.usrds.org/2005/ pres/09U_asn_05_autosomal_dominant_polycystic_kidney_dis pdf?zoom_highlight=autosomal+polycystic + perrone\#search $=\% 22$ aut osomal\%20polycystic\%20perrone\%22. Accessed August 20, 2018.
ClinicoEconomics and Outcomes Research

\section{Publish your work in this journal}

ClinicoEconomics and Outcomes Research is an international, peerreviewed open-access journal focusing on health technology assessment, pharmacoeconomics and outcomes research in the areas of diagnosis, medical devices, and clinical, surgical and pharmacological intervention. The economic impact of health policy and health systems
Submit your manuscript here: https://www.dovepress.com/clinicoeconomics-and-outcomes-research-jourat

organization also constitute important areas of coverage. The manuscript management system is completely online and includes a very quick and fair peer-review system, which is all easy to use. Visit http://www.dovepress.com/testimonials.php to read real quotes from published authors. 